\title{
In Silico Modelling of the Human Intestinal Microflora
}

\author{
Derk Jan Kamerman and Michael H.F. Wilkinson \\ Institute for Mathematics and Computing Science, \\ University of Groningen, P.O. Box 800, 9700 AV Groningen, The Netherlands
}

\begin{abstract}
The ecology of the human intestinal microflora and its interaction with the host are poorly understood. Though more and more data are being acquired, in part using modern molecular methods, development of a quantitative theory has not kept pace with this development. This is in part due to the complexity of the system, and to the lack of simulation environments in which to test what the ecological effect of a hypothetical mechanism of interaction would be, before resorting to laboratory experiments. The MIMICS project attempts to address this through the development of a system for simulation of the intestinal microflora. In this paper the design, possibilities and relevance of this simulator including an intestinal wall are discussed.
\end{abstract}

\section{Introduction}

This paper introduces an new field of application for computer simulation: the intestinal microflora and its interaction with the host. The human intestines harbour a complex microbial ecosystem, part bound to the intestinal wall or embedded in the mucus layer covering the wall, part inhabiting the lumen, especially of the large intestine. The intestinal microflora form a highly complex community of an estimated 400 species. However, since the greater part (60-85 $\%$ ) of the microscopically visible bacteria in faecal content cannot be cultured [8], the exact number of species, and even their nature and role in the ecosystem are not really known. The importance of the gut ecosystem stems from the fact that it is considered a first line of defence against invading pathogens [14], mediated through competition for substrate and wall binding sites, production of toxins by the resident microflora, etc. However, the precise mechanisms are poorly understood, particularly because of the culturability problems.

The Model Intestinal Microflora In Computer Simulation (MIMICS) project aims to provide a flexible means of modelling and simulating the intestinal microflora, and in particular those ecological processes which have consequences for the host. It will provide a platform for medical researchers to test hypotheses in silico before commencing with (costly) in vitro or in vivo experiments. The MIMICS project is inspired by a number of developments in medicine, concurrent with the increased availability of powerful computing facilities. The first development in medicine which is relevant to this project, and which indeed 
initiated it, is the rapid and alarming increase in antibiotics resistance [3]. In line with this there is a growing realization of the importance of the bacterial microflora inhabiting the human intestines in maintaining the hosts defenses, and in performing a large number of other important functions.

A separate development is the increased use of computer simulation techniques in many fields of medicine, both for teaching and research purposes. Though this trend has been clearly visible in fields such as surgery [6], in the field of the intestinal microflora this trend is much less pronounced, and only fairly recently have there been any significant developments [1].

The interest in modelling is growing in this particular community due to the increased volume of data on the intestinal ecology due to modern detection methods such as DNA probes [11]. The earliest computer models of the intestinal microflora date from the early eighties [4], but subsequently very little work has been done on modelling the intestinal microflora using computers and mathematics [2,7]; for a review see [1]. The MIMICS project is the first, and so far unique attempt to model the human intestinal microflora by means of parallel high performance computers $[1,16]$.

This paper describes the main tool: a large scale cellular automaton which can simulate both metabolic and transport processes in the human intestine including the intestinal wall. The software runs on the Cray J932 supercomputer of the Centre for High Performance Computing.

\section{Modelling the ecosystem}

\subsection{General approach}

In the intestinal ecosystem various processes take place e.g. flow, diffusion, peristalsis, metabolic processes, interactions between bacteria, and immunological processes. Given the complexity of this system we have to restrict ourselves to what Levins calls strategic modelling [10] aimed at qualitative understanding of the types of behaviour of a class of systems. This contrasts with what Levins calls tactical modelling aimed at quantitative prediction of the behaviour of a specific system e.g. a patient. Via this strategic modelling, using in silico simulation, we would like to obtain a better understanding of the ecosystem, and to propose ideas and suggestions for further in vivo, in vitro, and in silico studies regarding the mechanisms in and properties of the ecosystem. We aim to find out what behaviour can be found in theory, which part of the ecosystem is crucial for which kind of behaviour, and which parameters are influential. Furthermore, we aim to test consistencies of hypotheses, and to calibrate our model with in vivo observations.

Because of the many unknowns we have to keep our model simple, and it does not make sense to elaborate on certain knowns. For instance, as long as peristalsis is not implemented, it is not reasonable to model the flow in accordance to the sophisticated Navier Stokes equations. There is simply no gain in accuracy. Furthermore we want a flexible organization of the software, allowing easy insertion of extra types of metabolism, and allowing easy exchange of 
one way of modelling metabolism for another. This is why we computationally separate metabolism from flow and diffusion, in different subroutines.

\subsection{General model of the ecosystem}

The most prominent entity in the ecosystem of the human intestine is the intestine itself. We model it as a straight tube of varying diameter. From stomach to anus we distinguish: small intestine (length $4.98 \mathrm{~m}$, diameter $0.03 \mathrm{~m}$ ), caecum (length $0.12 \mathrm{~m}$, diameter $0.09 \mathrm{~m}$ ), and colon (length $0.90 \mathrm{~m}$, diameter $0.06 \mathrm{~m}$ ). Axially we divide the intestine into 100 parts of equal length, and radially we subdivide each part into 10 equi-volume conaxial cylinder-like volume elements. The geometry is shown in fig. 1 .

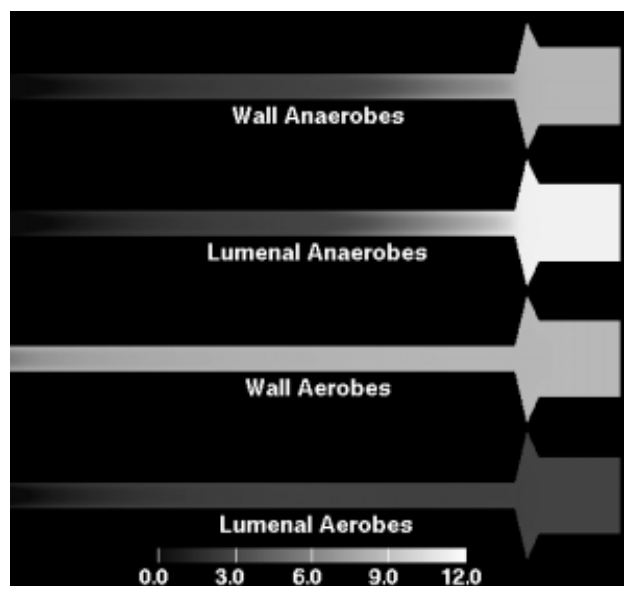

Fig. 1. The model intestinal geometry: four cross-sections of the intestine showing the concentrations of four bacterial species (in ${ }^{10} \log$ number bacteria / $\mathrm{ml}$ ).

The entities we distinguish next to the intestine are: food (one kind so far), oxygen, and various types of bacteria. The bacteria are subdivided based on their metabolism, e.g. anaerobes (do not use oxygen to grow) and aerobes (need oxygen to grow), and based on their ability to attach to the intestinal wall i.e. wall attaching bacteria and strictly lumenal bacteria.

The processes we distinguish in the ecosystem are metabolism and transport processes. The transport processes are transport into and out of the ecosystem and transport within the ecosystem. Transport into and out of the ecosystem includes: influx of food, oxygen and bacteria from the stomach, transport of food and oxygen through the intestinal wall, and efflux through the anus. Transport within the ecosystem consists of bulk motion modelled as laminar flow and turbulent and diffusive mixing modelled as diffusion with a rather large diffusion coefficient. 
In each time step, for which we use a value of about 5 minutes, we simulate metabolism, laminar flow, and diffusion consecutively. We thus separate computationally what in reality are simultaneously occurring processes.

\section{Metabolism in more detail}

In the metabolism subroutine we not only compute bacterial growth by metabolic activity and the corresponding decrease in food and oxygen concentrations, but also the increase and decrease of bacterium concentrations due to adhesion to and detachment from the intestinal wall. We do this for each of the volume elements separately, hence the computations can be done in parallel very well.

There is a large volume of literature devoted to modelling bacterial growth (see [9] for an overview). We have chosen one of the simplest but quite realistic models: the interacting Monod model, which is based on Michaelis-Menten-type kinetics [5]. The differential equations we use are solved by means of a fourthorder Runge-Kutta method, which can however easily be replaced by some other differential equation solver.

For each species or strain of bacteria $k$ with lumenal concentration $[X]_{k}(t)$ ( $m o l C \mathrm{~m}^{-3}$ ), the increase in bacterial concentration due to the metabolism of that bacterium in the lumen, denoted by $\mu_{l, k}(t)\left(\mathrm{mol} C \mathrm{~m}^{-3} \mathrm{~s}^{-1}\right)$, is modelled by the following differential equation

$$
\begin{aligned}
\mu_{l, k}(t)=\left(\left(\mu_{O_{2}, k} \frac{\left[O_{2}\right](t)}{K_{R, O_{2}, k}+\left[O_{2}\right](t)}\right.\right. & \left.+\mu_{a n, k}\right) \frac{[S](t)}{K_{S, k}+[S](t)} \\
& \left.-\kappa_{O_{2}, k} \frac{\left[O_{2}\right](t)}{K_{T, O_{2}, k}+\left[O_{2}\right](t)}-\mu_{b, k}\right)[X]_{k}(t) .
\end{aligned}
$$

In this equation, $[S](t)$ and $\left[O_{2}\right](t)$ represent the concentrations of food (or substrate) ( $\mathrm{mol} C \mathrm{~m}^{-3}$ ) and oxygen $\left(\mathrm{mol} \mathrm{m} \mathrm{m}^{-3}\right.$ ) in the volume element, as is experienced by the bacterium, i.e., the partial volume effect of bacteria is taken into account. This equation states that growth is based on the metabolism (first term), is reduced by killing due to toxicity of oxygen (second term) and by maintenance energy costs $\mu_{b, k}$. For the parameters in this equation and the following see table 1 .

The aerobic bacteria have no anaerobic metabolism $\left(\mu_{a n, k}=0\right)$, and have zero maximum oxygen kill rate $\kappa_{O_{2}, k}$. The anaerobic bacteria have an anaerobic metabolism (therefore $\mu_{a n, k}>0$ ) which is inhibited in the presence of oxygen $\left(\mu_{O_{2}, k}<0\right)$. On top of that the anaerobic bacteria we model suffer from losses due to the toxicity of oxygen $\left(\kappa_{O_{2}, k}>0\right)$. A more comprehensive discussion of the above Monod based equation can be found in [15].

Next to the distinction in anaerobes and aerobes, we have a distinction in strictly lumenal and wall attaching bacteria. In each volume element along the wall we model a wall population next to a lumenal population of the latter. It is essential that we give the bacteria which can attach to the wall a penalty for this extra ability. If not, it can easily be shown that these bacteria would outcompete the strict lumenals under all circumstances, which is both uninteresting 
Table 1. Parameters describing a bacterial metabolism, after [5]

\begin{tabular}{|c|c|c|c|c|c|c|}
\hline \multirow[b]{2}{*}{ Symbol } & \multirow[b]{2}{*}{ Meaning } & \multicolumn{2}{|c|}{ aerobes } & \multicolumn{2}{|c|}{ anaerobes } & \multirow[b]{2}{*}{ Units } \\
\hline & & lumenal & wall & lumenal & wall & \\
\hline$\mu_{\mathrm{O}_{2}}$ & $\begin{array}{l}\text { maximum specific } \\
\text { growth rate }\end{array}$ & $6.6 \cdot 10^{-4}$ & $6.0 \cdot 10^{-4}$ & $-1.1 \cdot 10^{-4}$ & $-1.0 \cdot 10^{-4}$ & $s^{-1}$ \\
\hline$\mu_{O_{2}, w}$ & as above, when wall bound & 0 & $5.4 \cdot 10^{-4}$ & 0 & $-0.9 \cdot 10^{-4}$ & $s^{-1}$ \\
\hline$\mu_{a n}$ & $\begin{array}{l}\text { maximum specific anaerobic } \\
\text { growth rate }\end{array}$ & 0 & 0 & $1.1 \cdot 10^{-4}$ & $1.0 \cdot 10^{-4}$ & $s^{-1}$ \\
\hline$\mu_{a n, w}$ & as above, when wall bound & \begin{tabular}{|l|}
0 \\
\end{tabular} & 0 & 0 & $0.9 \cdot 10^{-4}$ & $s^{-1}$ \\
\hline$\mu_{b}$ & maintenance costs & $1.0 \cdot 10^{-6}$ & $1.0 \cdot 10^{-6}$ & $1.0 \cdot 10^{-6}$ & $1.0 \cdot 10^{-6}$ & $s^{-1}$ \\
\hline$K_{S}$ & $\begin{array}{l}\text { food uptake saturation con- } \\
\text { stant }\end{array}$ & $2.0 \cdot 10^{+1}$ & $2.0 \cdot 10^{+1}$ & $2.0 \cdot 10^{+1}$ & $2.0 \cdot 10^{+1}$ & $m o l C m^{-3}$ \\
\hline$K_{R}$ & $\begin{array}{l}\text { respiratory oxygen uptake } \\
\text { rate constant }\end{array}$ & $1.0 \cdot 10^{-2}$ & $1.0 \cdot 10^{-2}$ & $1.0 \cdot 10^{-3}$ & $1.0 \cdot 10^{-3}$ & $\mathrm{~mol} \mathrm{O}_{2} \mathrm{~m}^{-3}$ \\
\hline$K_{T}$ & $\begin{array}{l}\text { toxic oxygen uptake rate con- } \\
\text { stant }\end{array}$ & $1.0 \cdot 10^{-2}$ & $1.0 \cdot 10^{-2}$ & $1.0 \cdot 10^{-3}$ & $1.0 \cdot 10^{-3}$ & $\mathrm{~mol} \mathrm{O}_{2} \mathrm{~m}^{-3}$ \\
\hline$\kappa_{\mathrm{O}_{2}}$ & maximum oxygen kill rate & 0.0 & 0.0 & $1.0 \cdot 10^{-6}$ & $1.0 \cdot 10^{-6}$ & $s^{-1}$ \\
\hline$\alpha_{\mathrm{O}_{2}}$ & yield of aerobic metabolism & 1.0 & 1.0 & 1.0 & 1.0 & \\
\hline$\alpha_{a n}$ & yield of anaerobic metabolism & 1.0 & 1.0 & 1.0 & 1.0 & \\
\hline$\alpha_{\kappa}$ & $\begin{array}{l}\text { fraction of oxygen killed bac- } \\
\text { teria returned as food }\end{array}$ & 1.0 & 1.0 & 0.5 & 0.5 & \\
\hline$\beta_{\mu}$ & $\begin{array}{l}\text { maximum respiratory oxygen } \\
\text { uptake rate }\end{array}$ & $1.5 \cdot 10^{-4}$ & $1.5 \cdot 10^{-4}$ & $1.0 \cdot 10^{-7}$ & $1.0 \cdot 10^{-7}$ & $s^{-1}$ \\
\hline$\beta_{\kappa}$ & $\begin{array}{l}\text { maximum oxygen uptake rate } \\
\text { due to toxic effect }\end{array}$ & 0.0 & 0.0 & $1.0 \cdot 10^{-7}$ & $1.0 \cdot 10^{-7}$ & $s^{-1}$ \\
\hline$p_{b}$ & $\begin{array}{l}\text { probability to attach to the } \\
\text { wall }\end{array}$ & 0 & 0.9 & 0 & 0.9 & \\
\hline$R_{\text {det }}$ & detachment rate & 0 & $3.0 \cdot 10^{-6}$ & 0 & $3.0 \cdot 10^{-6}$ & $s^{-1}$ \\
\hline$R_{a d h}$ & adhesion rate & 0 & $3.0 \cdot 10^{-5}$ & 0 & $3.0 \cdot 10^{-5}$ & $s^{-1}$ \\
\hline
\end{tabular}

and unrealistic. We penalize the bacterium with this wall attaching ability by giving it a smaller maximum specific growth rate, if only for the extra DNA it has to reproduce. Additionally, we penalize the bacterium while it is attached to the wall by giving it a smaller maximum specific growth rate again, because it has to express genes it otherwise would not, and it has a reduced effective surface area available for food and oxygen uptake.

The bacteria attached to the wall produce offspring according to (1), just as their lumenal counterparts do. Attached to the wall however, the growth rate $\mu_{w, k}(t)\left(\right.$ mol $\left.C m^{-2} s^{-1}\right)$ is lower, resulting in

$$
\begin{aligned}
\mu_{w, k}(t)=\left(\left(\mu_{O_{2}, w, k} \frac{\left[O_{2}\right](t)}{K_{R, O_{2}, k}+\left[O_{2}\right](t)}+\right.\right. & \left.\mu_{a n, w, k}\right) \frac{[S](t)}{K_{S, k}+[S](t)} \\
& \left.-\kappa_{O_{2}, k} \frac{\left[O_{2}\right](t)}{K_{T, O_{2}, k}+\left[O_{2}\right](t)}-\mu_{b, k}\right)[W]_{k}(t) .
\end{aligned}
$$

In this equation $[W]_{k}(t)$ is the concentration $\left(\mathrm{mol} \mathrm{C} \mathrm{m}^{-2}\right)$ of bacteria of strain $k$ attached to the wall, and the $w$ in $\mu_{O_{2}, w, k}$ and $\mu_{a n, w, k}$ denotes the reduction of the maximum specific growth rate of the bacterium in its wall attached state.

Some modifications are required however, in which we follow [7]. First of all, only part of this offspring will immediately attach to the wall, the rest will migrate into the lumen. A fraction $\eta_{w}(t)$ of the offspring of the wall attached bacteria emerges at a free place at the wall. The value of $\eta_{w}(t)$ in the volume 
element at hand depends on the number of bacteria already attached to the wall, and on the maximum number of bacteria that can attach in that place $\eta_{w}(t)=1-\frac{\sum_{k}[W]_{k}(t)}{[W]_{\max }}$. Each daughter cell of a bacterium attached to the wall, emerging at a free place, is assigned a probability $p_{b, k}$ to attach (or bind) to the wall. The rest of the offspring migrates into the lumen along the wall. This results in the following two equations: the increase in lumenal concentration of bacterium $k$ due to growth of the wall attached ones

$$
\frac{d[X]_{k}}{d t}(t) \mid \operatorname{metab}_{W_{k}}=\max \left\{0, \frac{A}{V}\left(1-p_{b, k} * \eta_{w}(t)\right) * \mu_{w, k}(t)\right\},
$$

and the increase in concentration at the wall of bacterium $k$ due to growth of wall attached bacteria

$$
\frac{d[W]_{k}}{d t}(t)\left|m e t a b=\mu_{w, k}(t)-\frac{V}{A} \frac{d[X]_{k}}{d t}(t)\right| \operatorname{metab}_{W_{k}} .
$$

In these equations an extra provision is taken in case the growth of the wall attached bacteria would become negative, in that case migration of negative amounts of offspring into the lumen must be prohibited. Furthermore, $V$ is the volume of the volume element and $A$ is the area of the intestinal wall in or along that volume element. Equations (1) and (3) combine to the differential equation for the bacterial concentration in a volume element due to bacterial growth,

$$
\frac{d[X]_{k}}{d t}(t)\left|\operatorname{metab}=\mu_{l, k}(t)+\frac{d[X]_{k}}{d t}(t)\right| \operatorname{metab}_{W_{k}}
$$

in which $\frac{d[X]_{k}}{d t}(t) \mid$ meta $b_{W_{k}}=0$ if the volume element is not along the intestinal wall.

Next to migration of offspring of wall attached bacteria, there is migration of lumenal bacteria to the wall (adhesion), and migration of wall attached bacteria into the lumen (detachment).

$$
\begin{aligned}
\frac{d[W]_{k}}{d t}(t) \mid \text { det } & =-R_{\text {det }, k}[W]_{k}(t) \\
\frac{d[X]_{k}}{d t}(t) \mid \text { det } & =\frac{A}{V} R_{\text {det }, k}[W]_{k}(t) \\
\frac{d[W]_{k}}{d t}(t) \mid a d h & =\eta_{w}(t) R_{a d h, k} * c *[X]_{k}(t) \\
\frac{d[X]_{k}}{d t}(t) \mid a d h & =-\frac{A}{V} \eta_{w}(t) R_{a d h, k} * c *[X]_{k}(t)
\end{aligned}
$$

The detachment rate $R_{\text {det }}$ and the adhesion rate $R_{a d h}$ are both measured in $s^{-1}$, and $c$ denotes a constant on the order of the radius of a bacterium, for the time being taken to be $0.5 * 10^{-6}(\mathrm{~m})$.

Bacterial growth, modelled in (1) and (2) is associated with uptake and release of oxygen and food. The next two equations balance the uptake and release 
of oxygen and food, by both the lumenal population and the wall population of the strains of bacteria present.

$$
\begin{aligned}
& \frac{d\left[O_{2}\right]}{d t}(t)=-\sum_{k}\left(\beta_{\mu, k} \frac{\left[O_{2}\right](t)}{K_{R, O_{2}, k}+\left[O_{2}\right](t)} \frac{[S](t)}{K_{S, k}+[S](t)}+\beta_{\kappa, k} \frac{\left[O_{2}\right](t)}{K_{T, O_{2}, k}+\left[O_{2}\right](t)}\right)[X]_{k}(t) \\
& -\frac{A}{V} \sum_{k}\left(\beta_{\mu, w, k} \frac{\left[O_{2}\right](t)}{K_{R, O_{2}, k}+\left[O_{2}\right](t)} \frac{[S](t)}{K_{S, k}+[S](t)}+\beta_{\kappa, k} \frac{\left[O_{2}\right](t)}{K_{T, O_{2}, k}+\left[O_{2}\right](t)}\right)[W]_{k}(t) \\
& \frac{d[S]}{d t}(t)=\sum_{k}\left(-\left(\frac{\mu_{O_{2}, k}}{\alpha_{O_{2}, k}} \frac{\left[O_{2}\right](t)}{K_{R, O_{2}, k}+\left[O_{2}\right](t)}+\quad \frac{\mu_{a n, k}}{\alpha_{a n, k}}\right) \frac{[S](t)}{K_{S, k}+[S](t)}\right. \\
& \left.+\alpha_{\kappa, k} \kappa_{O_{2}, k} \frac{\left[O_{2}\right](t)}{K_{T, O_{2}, k}+\left[O_{2}\right](t)}\right)[X]_{k}(t) \\
& +\frac{A}{V} \sum_{k}\left(-\left(\frac{\mu_{O_{2}, w, k}}{\alpha_{O_{2}, k}} \frac{\left[O_{2}\right](t)}{K_{R, O_{2}, k}+\left[O_{2}\right](t)}+\frac{\mu_{a n, w, k}}{\alpha_{a n, k}}\right) \frac{[S](t)}{K_{S, k}+[S](t)}\right. \\
& \left.+\alpha_{\kappa, k} \kappa_{O_{2}, k} \frac{\left[O_{2}\right](t)}{K_{T, O_{2}, k}+\left[O_{2}\right](t)}\right)[W]_{k}(t)
\end{aligned}
$$

\section{Flow and diffusion}

We model both flow and diffusion by difference equations. In contrast to metabolism, transport and diffusion take place not only within but also between volume elements. In modelling diffusion, we take a short enough time step to ensure that only diffusion between immediate neighbours need be considered. Difference equations can lead to numerical instabilities. In order to avoid those instabilities, we divide each volume element into four subdivisions, each bordering on a single subdivision of one of its neighbours, then analytically obtain the concentrations after diffusion in just each pair of bordering subdivisions of neighbouring volume elements, and after that we obtain the final concentrations in each volume element by taking the mean over the concentrations in the corresponding 4 constituent subdivisions.

We thus model flow and diffusion rather crudely, but the essential features (local mixing and overall flow) are preserved. The exact model used plus discussion can be found on the internet [15].

\section{Simulation results}

The first 90 days after colonization of a sterile intestine were simulated, with and without the possibility of attachment to the wall. The results with binding are shown in figures 1 and 2. The four bacterial species simulated have metabolic parameters as in table 1 . Further parameters such as flow rate, oxygen and food influx, etc. were set as in [16] 


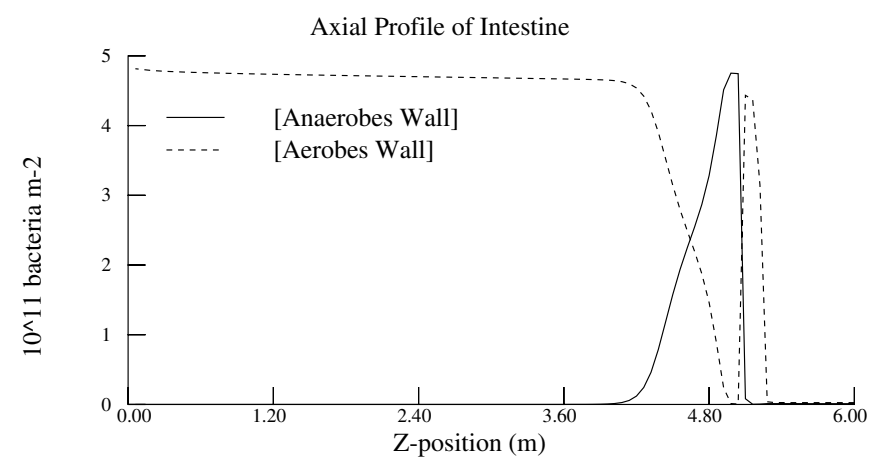

Fig. 2. Concentrations of anaerobic and aerobic bacteria attached to wall.

\section{Discussion}

In describing the results we will focus on those that give an impression of the tenability of modelling the human intestinal microflora in silico.

In our simulations we find that in initially sterile and oxygen rich bowels, as in newborns, first aerobic bacteria will prosper thereby reducing the oxygen concentration, and thus clearing the way for the anaerobic bacteria in the large intestine. The anaerobes, which initially cannot colonize at all due to the initial oxygen richness of the lumenal environment, will then prosper in the large intestine, reducing the oxygen concentration even further, making growth largely impossible for aerobic bacteria, and reducing the food concentration substantially. These findings are in line with in vivo studies, e.g. [12]. In the small intestine however, the anaerobes do not manage to colonize substantially due to the high flow rate of the bowel contents and the corresponding oxygen richness of the environment [16].

It is interesting that here we find a division of the ecosystem into two areas, an area where anaerobes are in the majority (large intestine), and an area where the aerobes are in the majority (small intestine). It is remarkable, that the concentrations of anaerobes $\left(3 * 10^{11}\right.$ bacteria $\left.g^{-1}\right)$ and aerobes $\left(5 * 10^{8}{\left.\text { bacteria } g^{-1}\right)}^{-}\right.$ in the large intestine, are in good agreement with what is found in vivo [8]. By contrast, though the numbers of bacteria in the small intestine is within the observed range, the ratio of anaerobes to aerobes is not. In our simulation we do not find many anaerobes though they are observed in vivo. The literature concerning these data is scarce however, and the accuracy is not very high. This difference in available data stems from the fact that measurements from the large intestine are obtained from faecal samples; samples from the small intestine require biopsies [8].

We find almost no bacterial growth in the colon. Most of the growth takes place in the caecum, which might be due to the light digestibility of the food we use. In the colon almost all of the growth which does occur, occurs along the 
intestinal wall. In vivo, Poulsen et al. [11] also observed a growing population along the intestinal wall, combined with a static one in the lumen of the colon of mice.

We find aerobes attached to the wall throughout the small intestine using the carrying capacity of the wall almost to the utmost. In addition, we find that when wall attachment is possible, the aerobes in the lumen of the small intestine are almost exclusively wall attaching bacteria, despite their slower growth. Otherwise, the aerobes in the lumen are almost exclusively strictly lumenal. This suggests wall attachment is an asset for aerobic bacteria.

Another thing we find in our simulations is that in contrast to the small intestine we find almost no bacteria attached to the wall in the distal colon. We do however find bacteria attached to the wall in the proximal colon. Note that these differences are not due to the wall model itself. In vivo, wall attachment of bacteria is found in the small intestine [8]. By contrast, in the large intestine of mice, Poulsen found bacteria in the mucus covering the wall, but not actually attached [11]. In our model the differences are due to different limits on growth. In the small intestine growth in the lumen is limited by both oxygen influx and dilution by the flow. Growth in the distal colon however, insofar existing, is limited by food and oxygen but not by dilution. Therefore bacteria in the small intestine that attach to the wall avoid dilution and thus a restriction on growth, finding a new restriction in the available space at the wall. Bacteria in the distal colon however do not escape a restriction on growth by attaching to the wall, but would become more restricted in their growth because of their penalty for being attached to the wall.

\section{Concluding remarks}

This modelling is a start. In due course more aspects will be modelled. As knowledge proceeds more accurate modelling can be employed, and as we want to uncover deeper laying facts more accurate modelling must be employed. So in time our model will grow in size and quality and more computing power will be needed.

In the near future we would like to add facultative bacteria, a mucus layer, a better geometry, different kinds of food, etcetera. In the not so near future we might integrate our software with in silico models of the immune system which are being built by others [13].

Next to biological adaptations we would like to make the software available for the community, therefore we would like to improve the interface with the user, but also to port the software from our shared memory vector machine to a cluster, making it available for a broader public.

Despite the simplicity of the modelling system used, several salient features of the intestinal microflora can be reproduced. Though tactical modelling may always be beyond our possibilities, this study shows strategic modelling is feasible. 


\section{References}

1. H. Boureau, L. Hartmann, T. Karjalainen, I. Rowland, and M. H. F. Wilkinson. Models to study colonisation and colonisation resistance. Microbial Ecology in Health and Disease, 12 suppl. 2:247-258, 2000.

2. M. E. Coleman, D. W. Dreesen, and R. G. Wiegert. A simulation of microbial competition in the human colonic ecosystem. Apllied and Environmental Microbiology, 62:3632-3639, 1996.

3. J. Davies. Bacteria on the rampage. Nature, 383:219-220, 1996.

4. R. Freter, H. Brickner, J. Fekete, M. M. Vickerman, and K. V. Carey. Survival and implantation of escherichia coli in the intestinal tract. Infection and Immunity, 39:686-703, 1983.

5. J. Gerritse, F. Schut, and J. C. Gottschal. Modelling of mixed chemostat cultures of an anaerobic bacterium Comamonas testosteroni, and an anaerobic bacterium Veilonella alcalescencs: comparison with experimental data. Applied and Environmental Microbiology, 58:1466-1476, 1992.

6. S.B. Issenberg, W. C. McGaghie, I. R. Hart, J. W. Mayer, J. M. Felner, E. R. Petrusa, R. A. Waugh, D. D. Brown, R. R. Safford, I. H. Gessner, D. L. Gordon, and G. A. Ewy. Simulation technology for health care professional skills training and assessment. JAMA, 282:861-866, 1999.

7. D. E. Kirschner and M. J. Blaser. The dynamics of helicobacter pylori infection in the human stomach. Journal of Theoretical Biology, 176:281-290, 1995.

8. B. Kleessen, E. Bezirtzoglou, and J. Mättö. Culture-based knowledge on biodiversity, development and stability of human gastrointestinal microflora. Microbial Ecology in Health and Disease, 12 suppl. 2:54-63, 2000.

9. A. L. Koch. The monod model and its alternatives. In A. L. Koch, J. A. Robinson, and G. A. Milliken, editors, Mathematical Modeling in Microbial Ecology, pages 6293. Chapman \& Hall, New York, 1998.

10. D. Levins. Evolution in a Changing Environment. Princeton University Press, Princeton, 1968.

11. L. K. Poulsen, F. Lan, C. S. Kristensen, P. Hobolth, S. Molin, and K. A. Krogfelt. Spatial distribution of Escherichia coli in the mouse large intestine inferred from rRNA in situ hybridization. Infection and Immunity, 62:5191-5194, 1994.

12. R. W. Schaedler, R. Dubos, and R. Costello. Association of germfree mice with bacteria isolated from normal mice. J. Exp. Med., 122:77-82, 1963.

13. H. B. Sieburg, J. A. McCutchan, O. Clay, L. Caballero, and J. J. Ostlund. Simulation of hiv-infection in artificial immune system. Physica D, 45:208-228, 1990.

14. D. van der Waaij, J. M. Berghuis-De Vries, and J. E. C. Lekkerkerk-Van der Wees. Colonization resistance of the digestive tract in conventional and antibiotic treated mice. Journal of Hygiene, 69:405-411, 1971.

15. M. H. F. Wilkinson. The mimics cellular automaton program design and performance testing. MIMICS Technical Report 1, Centre for High Performance Computing, University of Groningen, 1997. Available via http://www.cs.rug.nl/ michael/ under downloadable publications as pdf-file.

16. M. H. F. Wilkinson. Nonlinear dynamics, chaos-theory, and the "sciences of complexity": their relevance to the study of the interaction between host and microflora. In P. J. Heidt, V. Rusch, and D. van der Waaij, editors, Old Herborn University Seminar Monograph 10: New Antimicrobial Strategies, pages 111-130, HerbornDill, Germany, 1997. Herborn Litterae. 